\title{
A Non-Holonomic Systems Approach to Special Function Identities
}

\author{
Frédéric Chyzak \\ Algorithms Project \\ INRIA Paris-Rocquencourt \\ 78153 Le Chesnay (France) \\ frederic.chyzak@inria.fr
}

\author{
Manuel Kauers ${ }^{\dagger}$ \\ RISC-Linz \\ Johannes Kepler University \\ 4040 Linz (Austria) \\ mkauers@risc.jku.at
}

\author{
Bruno Salvy \\ Algorithms Project \\ INRIA Paris-Rocquencourt \\ 78153 Le Chesnay (France) \\ bruno.salvy@inria.fr
}

\begin{abstract}
We extend Zeilberger's approach to special function identities to cases that are not holonomic. The method of creative telescoping is thus applied to definite sums or integrals involving Stirling or Bernoulli numbers, incomplete Gamma function or polylogarithms, which are not covered by the holonomic framework. The basic idea is to take into account the dimension of appropriate ideals in Ore algebras. This unifies several earlier extensions and provides algorithms for summation and integration in classes that had not been accessible to computer algebra before.
\end{abstract}

\section{Categories and Subject Descriptors}

I.1.2 [Computing Methodologies]: Symbolic and Algebraic Manipulation-Algorithms

\section{General Terms}

Algorithms

\section{Keywords}

Symbolic Summation and Integration, D-Finite Functions

\section{INTRODUCTION}

In a classical article entitled "A holonomic systems approach to special functions identities" [16], Doron Zeilberger has shown that the theory of holonomic D-modules leads to algorithms for proving identities in large classes of special functions. In this setting, a function $f\left(x_{1}, \ldots, x_{n}\right)$ is represented by a system of linear differential equations with poly-

*F. C. and B. S.'s work was supported in part by the Microsoft Research - INRIA Joint Center.

†This work was carried out while M. K. was member of the Algorithms Project-Team at INRIA Paris-Rocquencourt. At RISC, M. K. is supported by FWF grant P19462-N18.

Permission to make digital or hard copies of all or part of this work for personal or classroom use is granted without fee provided that copies are not made or distributed for profit or commercial advantage and that copies bear this notice and the full citation on the first page. To copy otherwise, to republish, to post on servers or to redistribute to lists, requires prior specific permission and/or a fee.

ISSAC'09, July 28-31, 2009, Seoul, Korea.

Copyright 2009 ACM X-XXXXX-XX-X/XX/XX ...\$5.00. nomial coefficients that annihilate it. The function is "holonomic" when it possesses two important properties: (i) besides its defining system, $f$ can be specified by a finite number of initial conditions; (ii) the number of linearly independent functions among all $x_{1}^{m_{1}} \cdots x_{n}^{m_{n}} \frac{\partial^{k_{1}}}{\partial x_{1}^{k_{1}}} \cdots \frac{\partial^{k_{n}}}{\partial x_{n}^{k_{n}}}(f)$ with $m_{1}+\cdots+m_{n}+k_{1}+\cdots+k_{n} \leq N$ grows like $O\left(N^{n}\right)$. The first property has the consequence that many operations on holonomic functions reduce to linear algebra. It leads to closure properties under sum, product, and specialization. The second one is related to the notion of holonomic D-modules and opens the way to algorithms for definite integration and summation. For this, Zeilberger developed a general method called creative telescoping, for which he gave two algorithms: one for the general holonomic case and a faster one in the hypergeometric case.

Originally, the notion of a holonomic system is only defined for differential systems, but there are several ways of extending it to systems of difference (or $q$-difference) equations. Among those, we prefer the use of Ore algebras [6]. There, the first property above corresponds to zero-dimensional ideals, which are called $\partial$-finite. The same closure properties (sum, product, specialization) hold and can be performed by Gröbner bases computation. Chyzak [5] extended Zeilberger's fast hypergeometric creative telescoping to all $\partial$-finite ideals, termination being guaranteed inside Zeilberger's holonomic class.

Another direction of extension concerns functions or sequences that cannot be defined by a holonomic system or even a $\partial$-finite ideal. Majewicz [11] has given an algorithm that is able to produce Abel's summation identity

$$
\sum_{k=0}^{n}\left(\begin{array}{l}
n \\
k
\end{array}\right) i(k+i)^{k-1}(n-k+j)^{n-k}=(n+i+j)^{n}
$$

automatically and to find similar new identities. Kauers [9] has given a summation algorithm applicable to sums involving Stirling numbers and similar sequences defined by triangular recurrence equations. This algorithm finds, for instance,

$$
\sum_{k=0}^{n}(-1)^{m-k} k !\left(\begin{array}{c}
n-k \\
m-k
\end{array}\right) S_{2}(n+1, k+1)=E_{1}(n, m),
$$

where $S_{2}$ and $E_{1}$ refer to the Stirling numbers of second kind and the Eulerian numbers of first kind, respectively. A summation algorithm of Chen and Sun [4] is able to discover certain summation identities involving Bernoulli numbers $B_{n}$ 


\begin{tabular}{lll}
\hline \hline Operator & $\partial \cdot f(x)$ & $x \cdot f(x)$ \\
\hline Differentiation $\frac{d}{d x}$ & $f^{\prime}(x)$ & $x f(x)$ \\
Shift $S$ & $f(x+1)$ & $x f(x)$ \\
Difference $\Delta$ & $f(x+1)-f(x)$ & $x f(x)$ \\
$q$-Dilation $Q$ & $f(q x)$ & $x f(x)$ \\
Continuous $q$-difference & $f(q x)-f(x)$ & $x f(x)$ \\
$q$-Differentiation $\delta^{(q)}$ & $\frac{f(q x)-f(x)}{(q-1) x}$ & $x f(x)$ \\
$q$-Shift $S^{(q)}$ & $f(x+1)$ & $q^{x} f(x)$ \\
Discrete $q$-difference $\Delta(q)$ & $f(x+1)-f(x)$ & $q^{x} f(x)$ \\
Eulerian operator $\Theta$ & $x f^{\prime}(x)$ & $x f(x)$ \\
Mahlerian operator $M$ & $f\left(x^{b}\right)$ & $x f(x)$ \\
Divided differences & $\frac{f(x)-f(a)}{x-a}$ & $x f(x)$ \\
\hline \hline
\end{tabular}

Table 1: Some common Ore operators

or similar quantities, for example

$$
\sum_{k=0}^{m}\left(\begin{array}{l}
m \\
k
\end{array}\right) B_{n+k}=(-1)^{m+n} \sum_{k=0}^{n}\left(\begin{array}{l}
n \\
k
\end{array}\right) B_{m+k} .
$$

None of the quantities covered by these algorithms admits a definition via a $\partial$-finite ideal, but all three algorithms are based on principles that resemble those employed for holonomic systems and $\partial$-finite ideals. In each case, it turns out that the differential/difference equations defining the integrand/summand are of a form that permits to prove the existence of at least one non-trivial differential/difference equation for the integral/sum by a counting argument.

In this article, we give algorithms dealing with ideals of Ore algebras that are not $\partial$-finite. They generalize the algorithms known for the $\partial$-finite case and cover the extensions to non-holonomic functions discussed so far. Holonomy being lost, it is not always the case that creative telescoping can succeed. However, holonomy is only a sufficient condition. We show that by considering more generally the dimension of the ideals and another quantity that we call polynomial growth, it is possible to predict termination of a generalization of Chyzak's generalization of Zeilberger's fast algorithm. As special cases, we recover holonomic systems (dimension 0, polynomial growth 1), but also the special purpose algorithms mentioned above for Abel-type sums (dimension 2, polynomial growth 1), Stirling-number identities and summation identities about Bernoulli numbers (dimension 1, polynomial growth 1). In addition, we get for free a summation/integration algorithm that can deal with nonholonomic special functions such as the incomplete Gamma function $\Gamma(n, z)$, the Hurwitz zeta function $\zeta(n, z)$, polylogarithms $\operatorname{Li}_{n}(x), \ldots$ Examples are given in Section 4.4.

\section{ORE ALGEBRAS AND THEIR IDEALS}

Our motivation for using Ore algebras is the convenient polynomial representation of linear operators that they offer. Classical notions of commutative polynomial rings generalize to this setting. In this section, we recall without proof the basic definitions and facts we use (see $[6,8]$ and their references for proofs, details, and history).

\subsection{Ore Algebras}

\section{$\sigma$-derivations}

Let $A$ be a commutative algebra over a field $k$, and $\sigma$ an injective algebra endomorphism of $A$ that induces the identity on $k$. A $k$-linear endomorphism $\delta$ of $A$ is called a

\begin{tabular}{lccc}
\hline \hline Operator & $\sigma$ & $\delta$ & $\partial x$ \\
\hline Differentiation & $\mathrm{Id}$ & $\frac{d}{d x}$ & $x \partial+1$ \\
Shift $S$ & $S$ & 0 & $(x+1) \partial$ \\
Difference & $S$ & $\Delta$ & $(x+1) \partial+1$ \\
$q$-Dilation & $Q$ & 0 & $q x \partial$ \\
Cont. $q$-difference & $Q$ & $Q-\mathrm{Id}$ & $q x \partial+(q-1) x$ \\
$q$-Differentiation & $Q$ & $\frac{1}{(q-1) x}(Q-\mathrm{Id})$ & $q x \partial+1$ \\
$q$-Shift & $Q$ & 0 & $q x \partial$ \\
Discr. q-difference & $Q$ & $Q-\mathrm{Id}$ & $q x \partial+(q-1) x$ \\
Eulerian operator & $\mathrm{Id}$ & $x \frac{d}{d x}$ & $x \partial+x$ \\
Mahlerian operator & $M$ & 0 & $x^{b} \partial$ \\
Divided differences & $f \mapsto f(a) x \mapsto \frac{f(x)-f(a)}{x-a}$ & $a \partial+1$ \\
\hline \hline
\end{tabular}

Table 2: Corresponding skew-polynomial rings and their commutation rules

$\sigma$-derivation if it satisfies the skew Leibniz rule $\delta(u v)=$ $\sigma(u) \delta(v)+\delta(u) v$ for all $u$ and $v$ in $A$.

\section{Skew-polynomial rings}

The associative ring generated over $A$ by a new indeterminate $\partial$ and the relations $\partial u=\sigma(u) \partial+\delta(u)$ for all $u \in A$ is called a (left) skew-polynomial ring. It is denoted by $A[\partial ; \sigma, \delta]$. It does not have zero-divisors. It is called an extension of the skew-polynomial ring $B\left[\partial ; \sigma^{\prime}, \delta^{\prime}\right]$ when $B \subset A$, $\sigma^{\prime}=\left.\sigma\right|_{B}$ and $\delta^{\prime}=\left.\delta\right|_{B}$. In examples, we allow ourselves to use different symbols in place of $\partial$ for increased readability. In particular, we use $S_{n}, S_{k}$, etc. for denoting both the indeterminate $\partial$ and the corresponding $\sigma$ in shift algebras.

\section{Ore operators}

A skew polynomial $L \in A[\partial ; \sigma, \delta]$ acts on left $A[\partial ; \sigma, \delta]$ modules - in most of our applications, these are modules of functions, power series, or sequences. Solving then means finding an element $h$ in such a module such that $L \cdot h=0$. In this perspective, skew polynomials are called Ore operators.

For any $\lambda \in A$, the action $\partial: a \mapsto \partial \cdot a=\lambda \sigma(a)+\delta(a)$ turns the algebra $A$ itself into a left $A[\partial ; \sigma, \delta]$-module. For any $u, v$ in $A$, one has the product rule $\partial \cdot u v=\sigma(u) \partial \cdot v+\delta(u) v$.

Tables 1 and 2 illustrate some common types of Ore operators when $A=k[x]$, together with the values of $\sigma$ and $\delta$ that define the associated skew-polynomial ring.

In all these examples, $\sigma$ and $\delta$ can be written as $A$-linear combinations of $\partial$ and the identity. We call linear a skewpolynomial ring with this property.

\section{Ore algebras}

Ore algebras are a generalization of skew-polynomial rings well suited to the manipulation of multivariate special functions. Let $C(\boldsymbol{x})=C\left(x_{1}, \ldots, x_{m}\right)$ be a field of characteristic 0 of rational functions, $\left(\sigma_{1}, \ldots, \sigma_{n}\right)$ be $n C$-algebra morphisms of $C(\boldsymbol{x})$ commuting pairwise, and for each $i$, let $\delta_{i}$ be a $\sigma_{i}$-derivation, such that the $\delta_{i}$ 's commute pairwise and commute with the $\sigma_{j}$ 's when $j \neq i$. The Ore algebra $\mathbb{O}_{\boldsymbol{x}}=C(\boldsymbol{x})\langle\boldsymbol{\partial}\rangle$ is the associative $C(\boldsymbol{x})$-algebra generated by indeterminates $\boldsymbol{\partial}=\left\{\partial_{1}, \ldots, \partial_{n}\right\}$ modulo the relations

$$
\partial_{i} a=\sigma_{i}(a) \partial_{i}+\delta_{i}(a) \quad(a \in C(\boldsymbol{x})), \quad \partial_{i} \partial_{j}=\partial_{j} \partial_{i} .
$$

An Ore algebra $\mathbb{O}$ or an extension $A \otimes_{C(\boldsymbol{x})} \mathbb{O}$ of it is called linear when for each $i$, both $\sigma_{i}$ and $\delta_{i}$ can be expressed as $C(\boldsymbol{x})$-linear combinations of $\partial_{i}$ and the identity.

Given two Ore algebras $\mathbb{O}_{x}$ and $\mathbb{O}_{t}$, we write $\mathbb{O}_{x, t}$ or with an abuse of notation $C(\boldsymbol{x}, \boldsymbol{t})\left\langle\boldsymbol{\partial}_{\boldsymbol{x}}, \boldsymbol{\partial}_{\boldsymbol{t}}\right\rangle$ for $\mathbb{O}_{\boldsymbol{x}} \otimes_{C} \mathbb{O}_{\boldsymbol{t}}$. 


\subsection{Ideals}

We write $I \unlhd R$ to denote that $I$ is a left ideal in the ring $R$, and $I=\left\langle p_{1}, \ldots, p_{k}\right\rangle$ to denote that $I$ is generated by $p_{1}, \ldots, p_{k}$. The study of a function or sequence $f$ translates algebraically into the study of its annihilating ideal $\operatorname{ann}_{A} f$, i.e., the left ideal of operators in an appropriate algebra $A$ that annihilate $f$. (We also write ann $f$ when no ambiguity on $A$ can arise.) Computations concern finding generators of this ideal or, at least, of a sufficiently large subideal of it. This is in particular the case for creative telescoping that we study here. It computes an ideal annihilating the definite integral or sum of interest starting from a description of an ideal annihilating the summand or integrand.

\subsection{Gröbner Bases}

\section{Terms}

If $f=\sum c_{\alpha} \partial^{\alpha}$ is a polynomial, each $c_{\alpha} \partial^{\alpha}$ for which $c_{\alpha} \neq 0$ is called a term of $f, c_{\alpha}$ is its coefficient, $\partial^{\alpha}$ its monomial, and $\boldsymbol{\alpha}$ its exponent. The total degree of $f$ is the maximum $|\boldsymbol{\alpha}|$ over its terms, where we use the notation $|\boldsymbol{\alpha}|=\left|\left(\alpha_{1}, \ldots, \alpha_{n}\right)\right|=\alpha_{1}+\cdots+\alpha_{n}$. We also use $|S|$ for the cardinality of a set $S$, but this should not create confusion.

\section{Monomial orders}

A monomial order is a total order on the monomials that is compatible with the product and does not have infinite descending chains. A graded order is a monomial order such that $\partial^{\alpha}>\partial^{\beta}$ whenever $|\boldsymbol{\alpha}|>|\boldsymbol{\beta}|$.

\section{Gröbner bases}

For a given monomial order, the leading term of a polynomial $f$ is the term with largest monomial for that order. We write $\mathrm{E}(f)$ for its exponent in $\mathbb{N}^{n}$. The crucial property that lets the theory parallel that of the commutative case is that $\mathrm{E}(f g)=\mathrm{E}(f)+\mathrm{E}(g)$. The set $S_{I}:=\{\mathrm{E}(f), f \in I\}$ thus has the property $S_{I}=S_{I}+\mathbb{N}^{n}$. The complement of $S_{I}$ is a finite union of translates of coordinate subspaces. A Gröbner basis $G$ of $I$ is a set of generators $I$ such that $S_{I}$ is the sum of $\mathrm{E}(g)+\mathbb{N}^{n}$ over $g \in G$.

\subsection{Hilbert Dimension}

We write $R^{(s)}$ for the set of polynomials of an Ore algebra $R$ of total degree at most $s$. Then $I^{(s)}:=I \cap R^{(s)}$ is a vector space over $C(\boldsymbol{x})$. As in the commutative case, the Hilbert function of the ideal $I$ is defined by $\operatorname{HF}_{I}(s):=$ $\operatorname{dim}\left(R^{(s)} / I^{(s)}\right)=\operatorname{dim} R^{(s)}-\operatorname{dim} I^{(s)}$; for $s$ large enough this function is equal to a polynomial whose degree is called the (Hilbert) dimension of the ideal. We denote this integer $\operatorname{dim} I$, or $\operatorname{dim}_{R} I$ when we want to make the ambient ring explicit. A reference for the results of this section is [10].

Example 1 (D-Finite IDEAls). An ideal is $\partial$-finite if its dimension is 0 . This special class of ideals has been studied a lot from the computational point of view: in this case, the quotient $R / I$ is a finite-dimensional vector space, so that many techniques of linear algebra apply.

EXAmple 2 (Hypergeometric terms). An n-variate sequence $u_{m_{1}, \ldots, m_{n}}$ is a hypergeometric term if

$$
\frac{u_{m_{1}, \ldots, m_{i-1}, m_{i}+1, m_{i+1}, \ldots, m_{n}}}{u_{m_{1}, \ldots, m_{n}}} \in \mathbb{Q}\left(m_{1}, \ldots, m_{n}\right)
$$

for $i=1, \ldots, n$. If $\boldsymbol{S}=\left(S_{1}, \ldots, S_{n}\right), S_{i}$ representing the shift operator, the annihilating ideal of such a sequence in the algebra $\mathbb{Q}(\boldsymbol{m})\langle\boldsymbol{S}\rangle$ contains operators $S_{1}-r_{1}(\boldsymbol{m}), \ldots, S_{n}-$ $r_{n}(\boldsymbol{m})$. It is therefore $\partial$-finite and moreover, the dimension of the quotient as a vector space is 1 .

Example 3 (Stirling Numbers). Stirling numbers of the second kind, $S_{2}(n, k)$, satisfy the linear recurrence

$$
S_{2}(n, k)=S_{2}(n-1, k-1)+k S_{2}(n-1, k) .
$$

The ideal generated by this relation in $\mathbb{Q}(n, k)\left\langle S_{n}, S_{k}\right\rangle$ has dimension 1. Properties of the generating series imply that this is not a $\partial$-finite sequence, so that 1 is the lowest possible dimension to work with.

The dimension of an ideal can be computed from a Gröbner basis for a graded order: it is the largest dimension of coordinate subspaces of $\mathbb{N}^{n}$ that belong to the complement of the set of exponents of the leading terms [3, p. 449].

We note the following inequalities that also hold in this non-commutative context:

$$
\begin{aligned}
I \subset J \Rightarrow & \operatorname{dim} J \leq \operatorname{dim} I, \\
I \unlhd C(\boldsymbol{x}, \boldsymbol{t})\left\langle\boldsymbol{\partial}_{\boldsymbol{x}}, \boldsymbol{\partial}_{\boldsymbol{t}}\right\rangle \Rightarrow \operatorname{dim}_{C(\boldsymbol{x}, \boldsymbol{t})\left\langle\boldsymbol{\partial}_{\boldsymbol{x}}\right\rangle}\left(I \cap C(\boldsymbol{x}, \boldsymbol{t})\left\langle\boldsymbol{\partial}_{\boldsymbol{x}}\right\rangle\right) & \leq \operatorname{dim}_{C(\boldsymbol{x}, \boldsymbol{t})\left\langle\boldsymbol{\partial}_{\boldsymbol{x}}, \boldsymbol{\partial}_{\boldsymbol{t}}\right\rangle} I .
\end{aligned}
$$

The first one follows from the inclusion of the vector spaces $I^{(s)} \subset J^{(s)}$. The second inequality involves dimensions relative to two different ambient rings. It can be seen by considering the following vector spaces: $F=C(\boldsymbol{x}, \boldsymbol{t})\left\langle\boldsymbol{\partial}_{\boldsymbol{x}}, \boldsymbol{\partial}_{\boldsymbol{t}}\right\rangle^{(s)}$ contains $G=C(\boldsymbol{x}, \boldsymbol{t})\left\langle\boldsymbol{\partial}_{\boldsymbol{x}}\right\rangle^{(s)}$ and $H=I^{(s)}$. Then the inequality follows from $G /(G \cap H) \subset F / H$.

We also make use of the following properties of dimension.

Lemma 1. Let $I \unlhd \mathbb{O}_{\boldsymbol{x}}=C(\boldsymbol{x})\left\langle\boldsymbol{\partial}_{\boldsymbol{x}}\right\rangle$ with $\operatorname{dim} I=d$.

1. For any $\partial_{t} \subset \partial_{x},\left|\partial_{t}\right| \geq d+1 \Longrightarrow I \cap C(\boldsymbol{x})\left\langle\partial_{t}\right\rangle \neq\{0\}$.

2. There exists $\partial_{\boldsymbol{t}} \subset \partial_{\boldsymbol{x}}$ of cardinality d such that $I \cap C(\boldsymbol{x})\left\langle\boldsymbol{\partial}_{\boldsymbol{t}}\right\rangle=\{0\}$.

Proof. We sketch the proof which shows how the notions presented so far interact (exactly as in the commutative case). By definition of the Hilbert function,

$$
\begin{aligned}
& \operatorname{dim} I^{(s)}+\operatorname{dim} C(\boldsymbol{x})\left\langle\boldsymbol{\partial}_{\boldsymbol{t}}\right\rangle^{(s)} \\
= & \operatorname{dim} \mathbb{O}_{\boldsymbol{x}}^{(s)}+\operatorname{dim} C(\boldsymbol{x})\left\langle\boldsymbol{\partial}_{\boldsymbol{t}}\right\rangle^{(s)}-\mathrm{HF}_{I}(s) \\
= & \operatorname{dim} \mathbb{O}_{\boldsymbol{x}}^{(s)}+\left(\begin{array}{c}
\left|\boldsymbol{\partial}_{\boldsymbol{t}}\right|+s \\
s
\end{array}\right)-\operatorname{HF}_{I}(s) .
\end{aligned}
$$

The binomial is a polynomial in $s$ of degree $\left|\partial_{t}\right| \geq d+1$ with positive leading coefficient, so that for large enough $s$, it is larger than $\operatorname{HF}_{I}(s)$. The sum of the dimensions of the vector subspaces on the left-hand side is therefore larger than the dimension of $\mathbb{O}_{x}^{(s)}$ and thus they intersect nontrivially.

The second part follows by considering the exponents of leading terms. Now the combinatorial theory is exactly as in $\left[7\right.$, Ch. 9]. There exists a coordinate subspace of $\mathbb{N}^{n}$ of dimension $d$ in the complement set. This means that there exists a subset $\partial_{t} \subset \partial_{x}$ such that no monomial in $\partial_{t}$ is a leading term of an element of $I$. Thus there cannot be an element of $I$ in those variables only, as was to be proved. 


\section{CLOSURE PROPERTIES}

Our main result, Thm. 3 in the next section, generalizes the fact that holonomy is preserved under definite integration. First, we show how addition, multiplication, and action by $\partial$ behave with respect to dimension, generalizing the corresponding closure properties for $\partial$-finite ideals.

Theorem 1 (Closure Properties). Let $I_{1}, I_{2} \unlhd \mathbb{O}_{x}$ and let $f_{1}, f_{2}$ be annihilated by $I_{1}, I_{2}$, respectively. Then:

1. $\operatorname{dim} \operatorname{ann}\left(\partial \cdot f_{1}\right) \leq \operatorname{dim} I_{1}$ for all $\partial$ in $\left\{\boldsymbol{\partial}_{\boldsymbol{x}}\right\}$.

2. $\operatorname{dim} \operatorname{ann}\left(f_{1}+f_{2}\right) \leq \max \left(\operatorname{dim} I_{1}, \operatorname{dim} I_{2}\right)$.

3. If $f_{1}, f_{2}$ belong to the coefficient ring of a linear extension of $\mathbb{O}_{\boldsymbol{x}}$, then $\operatorname{dim} \operatorname{ann}\left(f_{1} f_{2}\right) \leq \operatorname{dim} I_{1}+\operatorname{dim} I_{2}$.

Proof. We show part 3. The arguments for parts 1 and 2 are similar and simpler.

Setting $k:=\operatorname{dim} I_{1}+\operatorname{dim} I_{2}+1$, it suffices to show that any $k$ elements $\partial_{1}, \ldots, \partial_{k}$ among $\partial$ are dependent modulo $\operatorname{ann}\left(f_{1} f_{2}\right)$.

Given a Gröbner basis for $I_{1}$ with respect to a graded order, each polynomial $P \in \mathbb{O}_{x}$ can be reduced to a normal form $\bar{P}$ such that $P \cdot f_{1}=\bar{P} \cdot f_{1}$ and $\operatorname{deg} \bar{P} \leq \operatorname{deg} P$. Moreover, by definition of the dimension, the set of all monomials in $\left\{\bar{P} \mid P \in R^{(s)}\right\}$ has cardinality growing like $O\left(s^{\operatorname{dim} I_{1}}\right)$. The same considerations hold for $f_{2}$.

By induction on the degree of $P$, the condition of a linear extension implies that $P \cdot\left(f_{1} f_{2}\right)$ rewrites as a linear combination of monomials $\left(\boldsymbol{\partial}^{\boldsymbol{\beta}} \cdot f_{1}\right)\left(\boldsymbol{\partial}^{\boldsymbol{\gamma}} \cdot f_{2}\right)$, with $|\boldsymbol{\beta}+\boldsymbol{\gamma}| \leq \operatorname{deg} P$. Moreover, we can assume that the monomials have been reduced to their normal forms and the inequalities still hold.

Let $s \geq 0$ and consider the following identities

$$
\partial_{1}^{\alpha_{1}} \cdots \partial_{k}^{\alpha_{k}} \cdot f_{1} f_{2}=\sum_{|\boldsymbol{\beta}| \leq s} \sum_{|\gamma| \leq s} u_{\boldsymbol{\alpha} ; \boldsymbol{\beta}, \boldsymbol{\gamma}}(\boldsymbol{x})\left(\boldsymbol{\partial}^{\boldsymbol{\beta}} \cdot f_{1}\right)\left(\boldsymbol{\partial}^{\gamma} \cdot f_{2}\right)
$$

$(|\boldsymbol{\alpha}| \leq s)$, where the sums on the right are constructed as above. Then the first sum actually ranges over a subset $\{\boldsymbol{\beta},|\boldsymbol{\beta}| \leq s\}$ of cardinality $O\left(s^{\operatorname{dim} I_{1}}\right)$ and the second one ranges over a subset $\{\gamma,|\gamma| \leq s\}$ of cardinality $O\left(s^{\operatorname{dim} I_{2}}\right)$. Thus there is a generating set of $O\left(s^{\operatorname{dim} I_{1}} s^{\operatorname{dim} I_{2}}\right)=O\left(s^{k-1}\right)$ monomials for all the summands. This implies that for $s$ large enough, there exists a nontrivial linear combination of the $O\left(s^{k}\right)$ polynomials in (2) of the form

$$
\sum_{|\boldsymbol{\alpha}| \leq s} w_{\boldsymbol{\alpha}}(\boldsymbol{x}) \partial_{1}^{\alpha_{1}} \cdots \partial_{k}^{\alpha_{k}} \cdot\left(f_{1} f_{2}\right)=0,
$$

as we wanted to show.

\section{Algorithm}

The proof gives an algorithm that computes generators of a subideal of the desired annihilating ideal, with a dimension that obeys the inequality. For increasing $s$, compute the normal forms of all monomials in $\partial$ of degree at most $s$, compute linear combinations between them (the kernel of the matrix $\left.\left(u_{\boldsymbol{\alpha} ; \boldsymbol{\beta}, \boldsymbol{\gamma}}\right)\right)$, and return these relations if they are sufficiently many to obtain the dimension of the theorem. For $\partial$-finite ideals, this returns the same result as the algorithms in [6]. Various optimizations are possible. The other closure operations are similar.
EXAMPLE 4. The sequence

$$
f_{n, m, k, l}=\left(\begin{array}{l}
n \\
k
\end{array}\right) S_{2}(k, l) S_{2}(n-k, m),
$$

is annihilated by an ideal of dimension at most 2. This follows from Thm. 1 by observing: (i) that $\left(\begin{array}{c}n \\ k\end{array}\right)$ is hypergeometric, thus $\partial$-finite, and thus annihilated by an ideal of dimension 0; (ii) that $S_{2}(k, l)$ and $S_{2}(n-k, m)$ are Stirlinglike (see Section 4.4) and thus annihilated by certain ideals of dimension 1. More specifically, the factors $\left(\begin{array}{l}n \\ k\end{array}\right), S_{2}(k, l)$, and $S_{2}(n-k, m)$ are annihilated by the ideals

$$
\begin{aligned}
& \left\langle(k-n-1) S_{n}+n+1,(k+1) S_{k}+k-n, S_{m}-1, S_{l}-1\right\rangle, \\
& \left\langle S_{n}-1, S_{k} S_{l}-(l+1) S_{l}-1, S_{m}-1\right\rangle, \\
& \left\langle S_{n} S_{k}-1,(m+1) S_{m} S_{k}+S_{k}-S_{m}, S_{l}-1\right\rangle,
\end{aligned}
$$$$
\text { respectively. The algorithm sketched above yields }
$$

$$
\begin{aligned}
I:= & \left\langle 1+n+(1+m)(1+n) S_{m}-(1-k+n) S_{n} S_{m},\right. \\
& (k-n) S_{m}+(1+k) S_{k} S_{l}+(1+k)(1+m) S_{k} S_{l} S_{m} \\
& +(1+l)(k-n) S_{l} S_{m}, 1+n+(1+l)(1+n) S_{l} \\
& \left.-(1+k) S_{k} S_{l} S_{n}\right\rangle \unlhd C(n, m, k, l)\left\langle S_{n}, S_{m}, S_{k}, S_{l}\right\rangle
\end{aligned}
$$

as an ideal of annihilators of $f_{n, m, k, l}$. It has dimension 2.

\section{CREATIVE TELESCOPING}

Creative telescoping is basically a combination of differentiation under the integral sign and integration by parts, or analogues for other operators. We now give it an algebraic interpretation. Our main theorem can be viewed as predicting cases when identities are bound to exist.

\subsection{Telescoping of an Ideal}

The heart of the method of creative telescoping translates algebraically into the notion of the telescoping of an ideal.

Definition 1. Let $I \unlhd \mathbb{O}_{\boldsymbol{x}, \boldsymbol{t}}=C(\boldsymbol{x}, \boldsymbol{t})\left\langle\boldsymbol{\partial}_{\boldsymbol{x}}, \boldsymbol{\partial}_{\boldsymbol{t}}\right\rangle$ be a left ideal. Assume $|\boldsymbol{t}|=\mid \partial_{\boldsymbol{t}} \overline{\mid}$. We define the telescoping of $I$ with respect to $\boldsymbol{t}=\left(t_{1}, \ldots, t_{k}\right)$ as the left ideal of $C(\boldsymbol{x})\left\langle\boldsymbol{\partial}_{\boldsymbol{x}}\right\rangle$

$$
T_{\boldsymbol{t}}(I):=\left(I+\partial_{t_{1}} \mathbb{O}_{\boldsymbol{x}, t}+\cdots+\partial_{t_{k}} \mathbb{O}_{\boldsymbol{x}, \boldsymbol{t}}\right) \cap C(\boldsymbol{x})\left\langle\boldsymbol{\partial}_{\boldsymbol{x}}\right\rangle .
$$

Definition 2. The variables $\partial_{t}=\left(\partial_{t_{1}}, \ldots, \partial_{t_{k}}\right)$ of the Ore algebra $\mathbb{O}_{x, t}$ are telescopable if there exist elements $a_{1}, \ldots, a_{k}$ in $C(\boldsymbol{x}, \boldsymbol{t})$ such that

$\delta_{t_{i}}\left(a_{i}\right) \in C(\boldsymbol{x}) \backslash\{0\} \quad$ and $\quad \sigma_{t_{i}}\left(a_{i}\right) \partial_{t_{j}}=\partial_{t_{j}} \sigma_{t_{i}}\left(a_{i}\right) \quad(j \neq i)$.

Note that this is a condition on the algebra, and does not depend on any specific ideal. In view of Table 1, this is not a strong restriction for our applications. For example, the differential operator $d / d t$ and the difference operator $\Delta_{t}$ are telescopable, with $a=t$, but the shift operator is not. This notion lets us generalize an idea of Wegschaider [14] used at the end of the proof of Thm. 3 below.

\subsection{Polynomial Growth}

In Thm. 3 below we give an upper bound for the dimension of $T_{t}(I)$, thus providing a termination criterion for the algorithms in Section 5. Our bound depends on the dimension of $I$ and on its "polynomial growth", defined as follows.

Definition 3. The left ideal $I \unlhd \mathbb{O}_{\boldsymbol{x}, t}$ has polynomial growth $p$ with respect to a given graded order if there exists a sequence of polynomials $P_{s}(\boldsymbol{x}, \boldsymbol{t}), s \in \mathbb{N}$, such that for 
any $\boldsymbol{\alpha}$ with $|\boldsymbol{\alpha}| \leq s$, the normal form of $P_{s}(\boldsymbol{x}, \boldsymbol{t}) \boldsymbol{\partial}^{\alpha}$ with respect to a Gröbner basis of I for the order has coefficients in $C(\boldsymbol{x})[\boldsymbol{t}]$ whose degrees with respect to $\boldsymbol{t}$ are $O\left(s^{p}\right)$. We say that I has polynomial growth $p$ when there is a graded order with respect to which it does.

If all the $\sigma$ 's are automorphisms, the polynomial growth is bounded by the dimension of the Ore algebra, $|\boldsymbol{x}|+|\boldsymbol{t}|$. But the interesting cases are those where the polynomial growth is smaller than that. For certain ideals of dimension 0, we get a better estimate in Thm. 2 below. For an arbitrary ideal in an arbitrary Ore algebra, we do not know how to determine its polynomial growth algorithmically yet.

ExAmPle 5. The basis at the end of Example 4 is a Gröbner basis with respect to a graded order. Inspection of its leading coefficients shows that the sequence

$$
P_{s}(n, m, k):=\prod_{|j| \leq s}(1+n-k+j)(1+k+j)^{2}
$$

satisfies the conditions of Definition 3. Since $\operatorname{deg} P_{s}=O(s)$, it follows that I has polynomial growth 1 .

Just before stating our result, we now give notation and a definition for algebras amenable to it.

Definition 4. Set $R=C(\boldsymbol{x})[\boldsymbol{t}]$ and let $R_{<n}$ be the set of elements $A \in R$ such that $\operatorname{deg}_{t} A \leq n$. A difference-differential algebra is an Ore algebra $\mathbb{O}_{\boldsymbol{x}, \boldsymbol{t}}$ such that for any $i$, either

- $\delta_{i}=0$ and, for any $u \in R, \operatorname{deg}_{t} \sigma_{i}(u)=\operatorname{deg}_{t} u$;

- or $\sigma_{i}=\operatorname{Id}$ and $\delta_{i}$ is a derivation such that, for any $u \in R, \operatorname{deg}_{t} \delta_{i}(u) \leq \nu+\operatorname{deg}_{t} u$,

$\nu \in \mathbb{N}$ being fixed. We set $\mathcal{S}$ and $\mathcal{D}$ to be the sets of $i$ of, resp., first and second types.

ThEOREM 2. Let $\mathbb{O}_{x, t}$ be a difference-differential algebra endowed with a graded ordering. Let $I \unlhd \mathbb{O}_{x, t}$ have dimension 0. Call $\phi$ the map sending any $A \in \mathbb{O}_{\boldsymbol{x}, t}$ to its normal form modulo $I$ w.r.t. the given graded ordering, and $\Gamma$ the (finite) set of monomials in normal form. Then, there exist $m, \ell \in \mathbb{N}$ and $L \in R_{\leq \ell}$ such that, for any $i$ and any $\boldsymbol{\beta} \in \Gamma$,

$$
\phi\left(\partial_{i} \partial^{\boldsymbol{\beta}}\right) \in \frac{1}{L} \sum_{\gamma \in \Gamma} R_{\leq m} \partial^{\gamma} .
$$

Define a sequence $\left(P_{s}\right)_{s \in \mathbb{N}}$ by $P_{0}=1$ and

$$
P_{s+1}:= \begin{cases}\operatorname{lcm}\left(P_{s}, \operatorname{lcm}\left(L \sigma_{i}\left(P_{s}\right)\right), P_{s} \operatorname{lcm}\left(L, Q_{s}\right)\right), & \text { if } \mathcal{D} \neq \emptyset \\ \operatorname{lcm}\left(P_{s}, \operatorname{lcm}\left(L \sigma_{i}\left(P_{s}\right)\right)\right), & \text { if } \mathcal{D}=\emptyset\end{cases}
$$

where $Q_{s}$ denotes the squarefree part of $P_{s}$ and the lcm in $\sigma_{i}$ is over all $i$.

If $\operatorname{deg}_{t} P_{s}=\Theta\left(s^{p}\right)$ for some integer $p>0$, then $I$ has polynomial growth $p$.

Before the proof, note that, by the definition of $\phi$, the sum in (3) is limited to $|\boldsymbol{\gamma}| \leq|\boldsymbol{\beta}|+1$. Moreover, the existence of a uniform $(L, m)$ is easily obtained in the zero-dimensional case, as the set of $(i, \boldsymbol{\beta})$ is then finite.

Proof. Introduce the sets $F_{s}=\left\{\boldsymbol{\partial}^{\boldsymbol{\alpha}},|\boldsymbol{\alpha}| \leq s\right\}$ for $s \in \mathbb{N}$. For $n \in \mathbb{N}$, define $R_{\leq n}[\Gamma]$ as $\sum_{\gamma \in \Gamma} R_{\leq n} \partial^{\gamma}$. For a fixed $s$, suppose there exists $P \in R$ and an integer $D$ for which

$$
\phi\left(F_{s}\right) \subset P^{-1} R_{\leq D}[\Gamma] .
$$

We look for homologues $P^{\prime}$ and $D^{\prime}$ for $F_{s+1}$. As $\phi$ satisfies

$$
\phi\left(F_{s+1}\right)=\phi\left(F_{s} \cup \bigcup_{i} \partial_{i} F_{s}\right) \subset \phi\left(F_{s}\right) \cup \bigcup_{i} \phi\left(\partial_{i} \phi\left(F_{s}\right)\right),
$$

we study $\phi\left(\partial_{i} A\right)$ for $A=P^{-1} U \boldsymbol{\partial}^{\boldsymbol{\beta}}$ when $U \in R_{\leq D}, \boldsymbol{\beta} \in \Gamma$, and $|\boldsymbol{\beta}| \leq s$. If $i \in \mathcal{S}$, then from $\delta_{i}=0$ follows $\partial_{i} A=$ $\sigma_{i}(P)^{-1} \sigma_{i}(U) \partial^{i} \partial^{\beta}$; therefore,

$$
\phi\left(\partial_{i} A\right) \in\left(L \sigma_{i}(P)\right)^{-1} R_{\leq D+m}[\Gamma] .
$$

Else, $i \in \mathcal{D}$ and from $\sigma_{i}$ being the identity follows $\partial_{i} A=$ $P^{-1} U \partial^{i} \boldsymbol{\partial}^{\boldsymbol{\beta}}+P^{-1} \delta_{i}(U) \boldsymbol{\partial}^{\boldsymbol{\beta}}+\delta_{i}\left(P^{-1}\right) U \boldsymbol{\partial}^{\boldsymbol{\beta}}$; thus, $\phi\left(\partial_{i} A\right)$ is in

$$
\frac{1}{L P} R_{\leq D+m}[\Gamma]+\frac{1}{L P} R_{\leq D+\ell+\nu} \partial^{\beta}+\frac{1}{P Q} R_{\leq D+D_{0}} \partial^{\beta}
$$

where $Q$ is the square-free part of $P$ and $D_{0}=\operatorname{deg}_{t} Q$. Defining $P^{\prime}$ as the $1 \mathrm{~cm}$ of $P, P \operatorname{lcm}(L, Q)$ if $\mathcal{D} \neq \emptyset$, and the $\sigma_{i}(P) L$ 's for $i \in \mathcal{S}$ yields $A, \phi\left(\partial_{i} A\right) \in\left(P^{\prime}\right)^{-1} R[\Gamma]$. Next, setting $D_{1}=\operatorname{deg}_{\boldsymbol{t}}\left(P^{\prime} / P Q\right)$ and $D_{2}=\operatorname{deg}_{\boldsymbol{t}}\left(P^{\prime} / L P\right)$, then $\Delta$ as the maximum of $\max \{m, \ell\}+D_{2}$ and $\max \{\ell+\nu+$ $\left.D_{2}, D_{0}+D_{1}\right\}$ if $\mathcal{D} \neq \emptyset$, finally $D^{\prime}=D+\Delta$ yields

$$
\phi\left(F_{s+1}\right) \subset\left(P^{\prime}\right)^{-1} R_{\leq D^{\prime}}[\Gamma] .
$$

Therefore, introducing the sequence $\left(P_{s}\right)$ defined in the theorem, we get by a first induction on $s$ that $\phi\left(F_{s}\right) \subset P_{s}^{-1} R[\Gamma]$. Next, under the additional assumption that $\operatorname{deg}_{t} P_{s}=\Theta\left(s^{p}\right)$ for some integer $p>0, P$ and $P^{\prime}$ in the proof above can be taken as $P_{s}$ and $P_{s+1}$, so that all of $D_{0}, D_{1}$, and $D_{2}$ are bounded by $\operatorname{deg} P_{s+1}-\operatorname{deg} P_{s}$, thus by $O\left(s^{p-1}\right)$. Therefore, by another induction

$$
\phi\left(F_{s}\right) \subset P_{s}^{-1} R_{\leq O\left(s^{p}\right)}[\Gamma],
$$

proving a polynomial growth $p$ for $I$.

In the uses of creative telescoping for summation, the identities (or ideals) are often stated in terms of shifts, while the operation of interest is a finite difference. However, it is possible to compute the polynomial growth in a differencedifferential algebra with shifts, and it will be the same when considered in an algebra with difference operators. This is the meaning of the following theorem, whose proof based on transporting graded orderings we omit.

Proposition 1. For a given difference-differential algebra $\mathbb{O}_{\boldsymbol{x}, \boldsymbol{t}}$ and indeterminates $\boldsymbol{u}=\left(u_{1}, \ldots, u_{k}\right)$, consider

$$
\begin{aligned}
\mathbb{O}_{\boldsymbol{x},(\boldsymbol{t}, \boldsymbol{u})} & =\mathbb{O}_{\boldsymbol{x}, \boldsymbol{t}} \otimes_{C} C(\boldsymbol{u})\left[\partial_{u_{1}} ; S_{u_{1}}, 0\right] \ldots\left[\partial_{u_{k}} ; S_{u_{k}}, 0\right] \quad \text { and } \\
\mathbb{O}_{\boldsymbol{x},(\boldsymbol{t}, \boldsymbol{u})}^{\prime} & =\mathbb{O}_{\boldsymbol{x}, \boldsymbol{t}} \otimes_{C} C(\boldsymbol{u})\left[\partial_{u_{1}} ; S_{u_{1}}, \Delta_{u_{i}}\right] \ldots\left[\partial_{u_{k}} ; S_{u_{k}}, \Delta_{u_{k}}\right],
\end{aligned}
$$

where $\Delta_{u_{i}}=S_{u_{i}}-\operatorname{Id}(i=1, \ldots, k)$. Let $\mu$ be the (bijective) left-C $(\boldsymbol{x}, \boldsymbol{t}, \boldsymbol{u})$-linear map sending each $\partial_{u_{i}}$ to $\partial_{u_{i}}+1$ and all the $\boldsymbol{\partial}_{\boldsymbol{x}}, \boldsymbol{\partial}_{\boldsymbol{t}}$ to themselves. Then an ideal $I \unlhd \mathbb{O}_{\boldsymbol{x},(\boldsymbol{t}, \boldsymbol{u})}$ and $\mu(I) \unlhd \mathbb{O}_{\boldsymbol{x},(\boldsymbol{t}, \boldsymbol{u})}^{\prime}$ have the same polynomial growth.

\subsection{Main Result}

Our main result is the following sufficient condition for creative telescoping. For simplicity, we state it for $|\boldsymbol{t}|=\left|\partial_{\boldsymbol{t}}\right|$.

Theorem 3 (Creative Telescoping). Let $I \unlhd \mathbb{O}_{\boldsymbol{x}, \boldsymbol{t}}=$ $C(\boldsymbol{x}, \boldsymbol{t})\left\langle\boldsymbol{\partial}_{\boldsymbol{x}}, \boldsymbol{\partial}_{\boldsymbol{t}}\right\rangle$ be an ideal of dimension $d$ and polynomial growth $p$. If $|\boldsymbol{t}|=\left|\boldsymbol{\partial}_{\boldsymbol{t}}\right|$ and the $\boldsymbol{\partial}_{\boldsymbol{t}}$ 's are telescopable then

$$
\operatorname{dim}_{\mathbb{O}_{\boldsymbol{x}}} T_{\boldsymbol{t}}(I) \leq d+(p-1)|\boldsymbol{t}|,
$$

whenever this bound is nonnegative. In particular, when the bound is smaller than $|\boldsymbol{x}|$, the ideal $T_{\boldsymbol{t}}(I)$ is non-trivial. 
Proof. If $d+(p-1)|\boldsymbol{t}| \geq|\boldsymbol{x}|$, there is nothing to show. Otherwise, by Lemma 1 , it is sufficient to show that any $k:=$ $d+(p-1)|\boldsymbol{t}|+1$ elements $\partial_{1}, \ldots, \partial_{k}$ in $\left\{\boldsymbol{\partial}_{\boldsymbol{x}}\right\}$ are dependent modulo $T_{t}(I)$.

Let $s \geq 0$ and consider the following set of members of $I$ from the definition of the polynomial growth of $I$ :

$$
P_{s}(\boldsymbol{x}, \boldsymbol{t}) \partial_{1}^{\alpha_{1}} \cdots \partial_{k}^{\alpha_{k}} \partial_{t}^{\alpha_{t}}-\sum_{|\boldsymbol{\beta}| \leq s} c_{\boldsymbol{\alpha}, \boldsymbol{\beta}}(\boldsymbol{x}, \boldsymbol{t}) \boldsymbol{\partial}^{\boldsymbol{\beta}}, \quad|\boldsymbol{\alpha}| \leq s .
$$

The coefficients $c_{\boldsymbol{\alpha}, \boldsymbol{\beta}}(\boldsymbol{x}, \boldsymbol{t})$ can be viewed as $C(\boldsymbol{x})$-linear combinations of monomials in $\boldsymbol{t}$ of degree $O\left(s^{p}\right)$. The ideal $I$ having dimension $d$, these sums in (4) for all $\boldsymbol{\alpha}$ such that $|\boldsymbol{\alpha}| \leq s$ actually range over a common subset of $\{\boldsymbol{\beta},|\boldsymbol{\beta}| \leq s\}$ of cardinality $O\left(s^{d}\right)$. Thus, there is a generating set of $O\left(s^{d+p|t|}\right)$ monomials in $\boldsymbol{t}, \boldsymbol{\partial}$ for all the summands. This implies that for $s$ large enough, there exists a nontrivial linear combination of the $O\left(s^{k+|t|}\right)$ polynomials in (4) of the form

$$
P_{s}(\boldsymbol{x}, \boldsymbol{t}) \sum_{|\boldsymbol{\alpha}| \leq s} C_{\boldsymbol{\alpha}}(\boldsymbol{x}) \partial_{1}^{\alpha_{1}} \cdots \partial_{k}^{\alpha_{k}} \boldsymbol{\partial}_{\boldsymbol{t}}^{\boldsymbol{\alpha}_{\boldsymbol{t}}}=: P(s) Q \in I .
$$

Multiplying by $1 / P_{s}(\boldsymbol{x}, \boldsymbol{t})$ shows that $Q \in I \cap C(\boldsymbol{x})\left\langle\boldsymbol{\partial}_{\boldsymbol{x}}, \partial_{\boldsymbol{t}}\right\rangle$. Now the operators $\partial_{t_{i}}$ commute with the coefficients of $Q$. Thus, $Q$ can be rewritten

$$
Q=R+\partial_{t_{1}} Q_{1}+\cdots+\partial_{t_{|t|}} Q_{|t|},
$$

with $R \in C(\boldsymbol{x})\left\langle\partial_{1}, \ldots, \partial_{k}\right\rangle \in T_{\boldsymbol{t}}(I)$. If $R \neq 0$, we have found the element of $T_{t}(I)$ we were looking for. Otherwise, since $Q \neq 0$, there exists a $Q_{i} \neq 0$. Since $\partial_{t_{i}}$ is telescopable, there is an element $a_{i} \in C(\boldsymbol{x}, \boldsymbol{t})$ such that

$$
\sigma_{t_{i}}\left(a_{i}\right) \partial_{t_{i}}=b_{i}+\partial_{t_{i}} a_{i}, \quad \sigma_{t_{i}}\left(a_{i}\right) \partial_{t_{j}}=\partial_{t_{j}} \sigma_{t_{i}}\left(a_{i}\right)
$$

for some $b_{i} \in C(x) \backslash\{0\}$. Multiplying (5) by $\sigma_{t_{i}}\left(a_{i}\right)$ yields

$$
I \ni \sigma_{t_{i}}\left(a_{i}\right) Q=b_{i} Q_{i}+\partial_{t_{i}} \tilde{Q}_{i}+\cdots+\partial_{t_{|t|}} \tilde{Q}_{|t|} .
$$

Now, $Q_{i} \neq 0$ can be rewritten as in (5). Repeating this process if necessary, we eventually reach a non-zero element of $T_{t}(I)$, as was to be proved.

\subsection{Examples}

\section{Proper hypergeometric sequences}

These are sequences with two indices that are hypergeometric as in Example 2 with the further constraint that they can be written

$$
u_{m, k}=Q(m, k) \frac{\prod_{i=1}^{u}\left(a_{i} m+b_{i} k+c_{i}\right) !}{\prod_{i=1}^{v}\left(u_{i} m+v_{i} k+w_{i}\right) !} \xi^{k},
$$

where $\xi \in \mathbb{C}, Q$ is a polynomial and the $a_{i}$ 's, $b_{i}$ 's, $u_{i}$ 's, $v_{i}$ 's are integers. A typical example is the binomial coefficient $\left(\begin{array}{c}m \\ k\end{array}\right)$. Since such a sequence is hypergeometric, a Gröbner basis of ann $u_{m, k}$ for any order is formed by the relations

$$
S_{m}-u_{m+1, k} / u_{m, k} \text { and } S_{k}-u_{m, k+1} / u_{m, k} .
$$

The normal form of $S_{m}^{s_{1}} S_{k}^{s_{2}}$ with respect to this basis is simply the rational function $u_{m+s_{1}, k+s_{2}} / u_{m, k}$. Since the $a_{i}, b_{i}, u_{i}, v_{i}$ 's are all integers, the common denominator of all these rational functions for $s_{1}+s_{2} \leq s$ has a degree that grows only linearly with $s[12]$, as it is bounded by

$$
\begin{aligned}
P_{s}(m, k)=Q(m, k) & \prod_{|j| \leq\left(\left|u_{i}\right|+\left|v_{i}\right|\right) s} \prod_{i}\left(u_{i} m+v_{i} k+w_{i}+j\right) \\
& \times \prod_{|j| \leq\left(\left|a_{i}\right|+\left|b_{i}\right|\right) s} \prod_{i}\left(a_{i} m+b_{i} k+c_{i}+j\right) .
\end{aligned}
$$

In our terminology, the corresponding annihilating ideal has polynomial growth 1, by Thm. 2 and Prop. 1. Further generalizations to the multivariate and $q$-cases can also be considered. Thm. 3 (with $d=0, p=1,|\boldsymbol{t}|=1, n=2$ ) generalizes the result that creative telescoping applies to proper hypergeometric sequences.

\section{General hypergeometric sequences}

Not every hypergeometric sequence is proper. For example,

$$
u_{m, k}=\frac{1}{m k+1}\left(\begin{array}{c}
2 m-2 k-1 \\
m-1
\end{array}\right)
$$

is not. By a criterion of Abramov [1], creative telescoping fails on this example, i.e., $T_{t}(I)=\{0\}$. This phenomenon is well consistent with our theorem, because the nonlinear factor in the denominator implies that the annihilating ideal $I$ has polynomial growth 2: a possible choice for $P_{s}$ is

$$
P_{s}(m, k)=\prod_{i+j \leq s}((m+i)(k+j)+1) \times \prod_{|i| \leq 3 s}(m-2 k+i),
$$

whose degree is quadratic in $s$, hence the polynomial growth by Thm. 2 and Prop. 1 . Thm. 3 (with $d=0, p=2,|\boldsymbol{t}|=$ $1, n=2$ ) implies the trivial bound $\operatorname{dim} T_{t}(I) \leq 1$, which is reached in this example.

\section{Holonomic Functions}

This notion was popularized for special functions in [16]. The technical definition is related to holonomic D-modules. Thm. 2 implies that it is sufficient to consider $\partial$-finite ideals, as was shown first by Takayama [13].

Proposition 2. Let $I \unlhd \mathbb{O}_{\boldsymbol{x}, \boldsymbol{t}}=C(\boldsymbol{x}, \boldsymbol{t})\left\langle\boldsymbol{\partial}_{\boldsymbol{x}, \boldsymbol{t}}\right\rangle$ be a left ideal of dimension 0 of the differential Ore algebra $\left(\sigma_{i}=\mathrm{Id}\right.$, $\left.\delta_{i}=d / d x_{i}\right)$. Then I has polynomial growth 1 .

Proof. The crucial property is that in the differential setting, the leading coefficient of an element does not change upon multiplication by some $\partial_{i}$ (see Table 2). The result is a corollary of Thm. 2, which is applicable since $I$ has dimension 0 . The polynomials $P_{s}$ defined in the theorem here take the form $P_{s}=L^{s}$, having degree $O\left(s^{1}\right)$ and thereby proving a polynomial growth 1 .

Put together, Prop. 2 and Thm. 3 imply $\operatorname{dim} T_{t}(I)=0$, and thus recover the celebrated closure of holonomic functions under definite integration as a special case.

\section{Stirling-like sequences}

This notion was introduced in [9]. A sequence is called Stirling-like if it is annihilated by an ideal of operators

$$
\left\langle u+v S_{k}^{\alpha} S_{m_{1}}^{\beta}+w S_{k}^{\gamma} S_{m_{1}}^{\delta}, s_{2}+t_{2} S_{m_{2}}, \ldots, s_{n}+t_{n} S_{m_{n}}\right\rangle,
$$

where $u, v, w, s_{2}, \ldots, s_{n}, t_{2}, \ldots, t_{n}$ are polynomials that split into integer-linear factors, and $\alpha, \beta, \gamma, \delta$ are integers subject to a certain nondegeneracy condition. A typical example is $\left(\begin{array}{c}m \\ k\end{array}\right) S_{2}(k, m)$, where $S_{2}$ refers to the Stirling numbers of 
the second kind. It was shown in [9] that such ideals lead to non-trivial creative telescoping relations whenever $n \geq 2$. These ideals have dimension 1 and polynomial growth 1 , so our theorem (with $d=1, p=1,|\boldsymbol{t}|=1, n \geq 3$ ) includes this result as a special case.

\section{Abel-type sequences}

This notion was introduced in [11]. A sequence is called Abel-type if it can be written in the form

$$
u_{m, k}(k+r)^{k}(m-k+s)^{m-k} \frac{r}{k+r}
$$

for some proper hypergeometric term $u_{m, k}$. The annihilating ideals of such sequences can be written

$$
\left\langle a S_{m} S_{k}-b S_{r}, c S_{m}-d S_{s}\right\rangle
$$

for certain polynomials $a, b, c, d$. It was shown in [11] that such ideals lead to non-trivial creative telescoping relations. These ideals have dimension 2 , and if $u_{m, k}$ is as in (6), then

$$
P_{s}(m, r, s, k)=\tilde{P}_{s}(m, k) \times \prod_{|j| \leq s}(k+r+j)(m-k+j),
$$

with $\tilde{P}_{s}(m, k)$ being the polynomial sequence stated above for proper hypergeometric terms, justifies that their polynomial growth is 1 , so our theorem (with $d=2, p=1,|\boldsymbol{t}|=1$, $n=4$ ) includes this result as a special case, too.

\section{Bernoulli examples}

Chen and Sun [4] do not give a formal description of the class of summands to which their algorithm is applicable. Most of their examples concern sums with summands of the form $h_{k, m_{1}, m_{2}} B_{a k+b m_{1}+c m_{2}}$ where $h$ is a hypergeometric term, $B$ refers to Bernoulli numbers, Bernoulli polynomials, Euler numbers, or Euler polynomials, and $a, b, c$ are specific integers. The annihilating ideals of such objects have dimension 1 , and, if $h$ is proper hypergeometric, polynomial growth 1. In this case, our theorem guarantees the success of creative telescoping.

\section{Further examples}

Our theorem also extends to sequences and functions for which no special purpose summation or integration algorithm has been formulated so far. For instance, the sequence $f_{n, m, k, l}$ considered in Examples 4 and 5 is not Stirling-like, but only annihilated by an ideal of dimension 2 and polynomial growth 1. Our Theorem predicts the existence of creative telescoping relations (cf. also Examples 6 and 7 below). We list some further identities from the literature that were previously considered inaccessible to computer algebra, but that can be proven with creative telescoping. In all the following identities, the integrand is not holonomic, but annihilated by an ideal of dimension 1 and polynomial growth 1 , so our theorem predicts a priori that relations for the integral must exist.

$$
\begin{aligned}
& \int_{0}^{\infty} x^{k-1} \zeta(n, \alpha+\beta x) d x=\beta^{-k} B(k, n-k) \zeta(n-k, \alpha) \\
& \int_{0}^{\infty} x^{\alpha-1} \operatorname{Li}_{n}(-x y) d x=\frac{\pi(-\alpha)^{n} y^{-\alpha}}{\sin (\alpha \pi)} \\
& \int_{0}^{\infty} x^{k-1} \exp (x y) \Gamma(n, x y) d x=\frac{\pi y^{-k}}{\sin ((n+k) \pi)} \frac{\Gamma(k)}{\Gamma(1-n)},
\end{aligned}
$$

$$
\int_{0}^{\pi} \cos (n x-z \sin x) d x=\pi J_{n}(z) .
$$

In these identities, $B$ refers to the Beta-function, $\zeta$ to the Hurwitz zeta function, $\mathrm{Li}_{n}$ to the $n$th polylogarithm, $\Gamma$ to the incomplete Gamma function, and $J_{n}$ to the $n$th Bessel function. Greek letters refer to parameters, $n, m, k$ are discrete variables, $x, y, z$ are continuous ones. Note that the polylogarithm, while holonomic for each specific $n$, is not even $\partial$-finite when $n$ is "symbolic". Also, the integrand of the last integral, despite being elementary, is not holonomic.

All these identities are proven by computing operators annihilating both sides, by making use of closure properties for sums, products or $\partial$ (Section 3) and by creative telescoping in the case of definite sums and integrals (Section 5 below). If the operators found in this way generate an ideal of dimension 0 , then proving the identity reduces to verifying a finite number of initial values. For higher dimensional ideals, the number of initial values to be checked may be infinite. For example, for completing the proof of the first integral identity above, it remains to check the identity for $k=1$ and all $n \geq 0$ :

$$
\int_{0}^{\infty} \zeta(n, \alpha+\beta x) d x=\frac{1}{\beta(n-1)} \zeta(n-1, \alpha),
$$

an identity not much easier than the original one. In many instances, however, the identities to be verified as initial conditions are trivial.

\section{ALGORITHMS FOR SUMMATION AND INTEGRATION}

\subsection{A Fasenmyer-Style Algorithm}

The first algorithmic approach to symbolic summation goes back to Fasenmyer and was formulated for the summation of hypergeometric terms [12]. The following generalized version of her algorithm is applicable to any given annihilating ideal in an Ore algebra.

The algorithm follows from the proof of Thm. 3. Given an ideal $I \unlhd C(\boldsymbol{x}, \boldsymbol{t})\left\langle\boldsymbol{\partial}_{\boldsymbol{x}}, \boldsymbol{\partial}_{t}\right\rangle$, the algorithm searches for elements of $I \cap \bar{C}(\boldsymbol{x})\left\langle\boldsymbol{\partial}_{\boldsymbol{x}}, \boldsymbol{\partial}_{\boldsymbol{t}}\right\rangle$ (called $\boldsymbol{t}$-free operators). Proceeding by increasing total degree, it makes an ansatz with undetermined coefficients for such an operator, reduces it to normal form with respect to a Gröbner basis for $I$, brings the normal form to a common denominator, and then compares the coefficients with respect to the $\boldsymbol{t}$ 's of the numerator to zero. This gives a linear system of equations over $C(\boldsymbol{x})$ for the undetermined coefficients, which is then solved. If the system has no solution, the procedure is repeated with a larger total degree. If a solution is found, it leads to an operator that can be written in the form (5)

$$
A+\partial_{t_{1}} B_{1}+\cdots+\partial_{t_{|t|}} B_{|t|} \in I
$$

where $0 \neq A \in T_{t}(I)$, by following the steps at the end of the proof that ensure $A \neq 0$. The procedure can be repeated to search for further operators in $T_{\boldsymbol{t}}(I)$ until all operators found generate an ideal of the dimension predicted by Thm. 3 .

EXAmple 6. For the ideal I from Example 4, with $k$ as summation variable, this algorithm discovers a first operator for total degree 4 that may be written in the form

$$
\begin{aligned}
S_{m}+S_{l}+(2+l+m) S_{l} S_{m}-S_{l} S_{m} S_{n} \\
\quad+\left(S_{k}-1\right)\left((m+1) S_{m} S_{l}-S_{m} S_{n} S_{l}+S_{l}\right) .
\end{aligned}
$$


As a consequence, we find

$$
A:=S_{m}+S_{l}+(2+l+m) S_{l} S_{m}-S_{l} S_{m} S_{n} \in T_{k}(I) .
$$

Therefore $A$ belongs to the annihilating ideal of the sum

$$
\sum_{k}\left(\begin{array}{l}
n \\
k
\end{array}\right) S_{2}(k, l) S_{2}(n-k, m) .
$$

This proves that the sum is equal to $\left(\begin{array}{c}l+m \\ l\end{array}\right) S_{2}(n, l+m): A$ also belongs to the annihilating ideal of that quantity, and it agrees with the sum for $n=0$ and arbitrary $l$ and $m$.

\subsection{A Zeilberger-Style Algorithm}

Zeilberger's "fast algorithm" was originally formulated for summation of proper hypergeometric terms only [15], then a differential analog was given for hyperexponential functions by Almkvist and Zeilberger [2]. This was later extended by Chyzak [5] to an integration/summation algorithm for arbitrary $\partial$-finite functions, with termination guaranteed in the holonomic case. We extend this algorithm further to the case of integrands or summands defined by arbitrary annihilating ideals.

The fast approach is applicable only for single sums or integrals, i.e., if $|\boldsymbol{t}|=1$. (Refer however to [5, Sec. 3.3] for an iterated treatment of summations and integrations.) Let $I \unlhd$ $C(\boldsymbol{x}, t)\left\langle\boldsymbol{\partial}_{\boldsymbol{x}}, \partial_{t}\right\rangle$ be given by a Gröbner basis $G$ with respect to a graded order. The algorithm searches for operators $A+\partial_{t} B \in I$ with $A \in C(\boldsymbol{x})\left\langle\boldsymbol{\partial}_{\boldsymbol{x}}\right\rangle$ and $B \in C(\boldsymbol{x}, t)\left\langle\boldsymbol{\partial}_{\boldsymbol{x}}, \partial_{t}\right\rangle$. Proceeding by increasing total degree, it makes an ansatz for both $A$ and $B$ and computes the normal form of $Q:=$ $A+\partial_{t} B$ with respect to $G$. Without loss of generality, only irreducible terms need to be included in the ansatz for $B$. In order for $Q$ to belong to $I$, it is necessary and sufficient that all the coefficients in the normal form of $Q$ be zero. Comparing them to zero leads to a system of first order linear functional equations (a "coupled system"), which is then solved. While in the $\partial$-finite case this system is always square, in the case of positive dimension it may be rectangular, owing to extraneous equations potentially being introduced by irreducible terms in $\partial_{t} B$ of degree $d+1$. However, it is always possible to separate the equations into a square coupled system (that can be solved in a first step as in Chyzak's algorithm) and a system with additional linear algebraic constraints (that can be accommodated in a second step by computing suitable linear combinations of the solutions obtained in the first step). Any solution $A+\partial_{t} B \in I$ found in this way gives rise to an element $A \in T_{t}(I)$. The procedure may be repeated until the elements of $T_{t}(I)$ found in this way generate an ideal in $C(\boldsymbol{x})\left\langle\boldsymbol{\partial}_{\boldsymbol{x}}\right\rangle$ whose dimension matches the dimension predicted by Thm. 3 .

EXAMPLE 7. Applying the algorithm to the ideal I of Example 4 with respect to $k$, we obtain a coupled system of size $5 \times 14$ when $A$ and $B$ are assumed to have total degree 2 and 1 , respectively. This system has no solution. For total degrees 3 and 2, respectively, the obtained system is of size $14 \times 28$ and has the nontrivial solution

$$
\begin{aligned}
& A=S_{m}+S_{l}+(2+l+m) S_{l} S_{m}-S_{l} S_{m} S_{n}, \\
& B=\frac{k(k+1)}{k^{2}-1-n-k n} S_{l}+\frac{(m+1) k}{k-n-1} S_{m} S_{l},
\end{aligned}
$$

hence $A+\left(S_{k}-1\right) B \in I$, and $A \in T_{k}(I)$.

\section{FINAL COMMENTS}

We have seen that algorithms for special functions doing computations in Ore algebras are not restricted to special functions with $\partial$-finite annihilating ideals. Instead, both closure properties algorithms and algorithms for definite integration and summation can be formulated for arbitrary ideals. The treatment could be extended further by including, for instance, ideals of Laurent Ore algebras.

Our generalized algorithms rely on the notion of ideal dimension as well as on the notion of polynomial growth we introduced in Definition 3. According to this definition, the polynomial growth depends on the monomial order imposed on the underlying algebra. Future research will focus on reducing the notion of polynomial growth to an intrinsic property of the ideal at hand, as well as to devising an algorithm for computing the polynomial growth.

\section{REFERENCES}

[1] S. A. Abramov. Applicability of Zeilberger's algorithm to hypergeometric terms. In ISSAC, pages 1-7, 2002.

[2] G. Almkvist and D. Zeilberger. The method of differentiating under the integral sign. J. Symb. Comp., 10(6):571-591, 1990.

[3] T. Becker and V. Weispfenning. Gröbner bases, volume 141 of Grad. Texts in Math. Springer, 1993.

[4] W. Y. C. Chen and L. H. Sun. Extended Zeilberger's algorithm for identities on Bernoulli and Euler polynomials. Technical Report 0810.0438, arXiv, 2008.

[5] F. Chyzak. An extension of Zeilberger's fast algorithm to general holonomic functions. Discrete Mathematics, 217(1-3):115-134, 2000.

[6] F. Chyzak and B. Salvy. Non-commutative elimination in Ore algebras proves multivariate holonomic identities. J. Symb. Comp., 26(2):187-227, 1998.

[7] D. Cox, J. Little, and D. O'Shea. Ideals, varieties, and algorithms. Springer-Verlag, 1996.

[8] A. Kandri-Rody and V. Weispfenning. Non-commutative Gröbner bases in algebras of solvable type. J. Symb. Comp., 9(1):1-26, 1990.

[9] M. Kauers. Summation algorithms for Stirling number identities. J. Symb. Comp., 42(10):948-970, 2007.

[10] M. V. Kondratieva, A. B. Levin, A. V. Mikhalev, and E. V. Pankratiev. Differential and Difference Dimension Polynomials. Kluwer, 1999.

[11] J. E. Majewicz. WZ-style certification and sister Celine's technique for Abel-type identities. $J$. Difference Eqs and Appl., 2:55-65, 1996.

[12] M. Petkovšek, H. Wilf, and D. Zeilberger. $A=B$. AK Peters, Ltd., 1997.

[13] N. Takayama. An approach to the zero recognition problem by Buchberger algorithm. J. Symb. Comp., 14:265-282, 1992.

[14] K. Wegschaider. Computer generated proofs of binomial multi-sum identities. Master's, RISC, 1997.

[15] D. Zeilberger. A fast algorithm for proving terminating hypergeometric identities. Discrete Mathematics, 80(2):207-211, 1990.

[16] D. Zeilberger. A holonomic systems approach to special functions identities. J. Comp. and Applied Maths, 32(3):321-368, 1990. 